\title{
DEALTERNATING NUMBERS AND CLASSICAL LINK INVARIANTS
}

\author{
Myung Jae Kim, Dong-Hee Lee, and Dongseok Kim
}

\begin{abstract}
Dealternating numbers and alternation numbers measure the distance between the link and an alternating links. In the present article, we show that classical link invariants; the determinant, signature and Alexander polynomial can not detect the almost alternativity of links.
\end{abstract}

\section{Introduction}

A link $L$ is a disjoint union of circles embedded in three dimensional sphere $\mathbb{S}^{3}$, and a knot $K$ is a link with only one component. A link diagram $D_{L}$ is a projection of the link $L$ onto a plane as the link casting a shadow on the wall which is one-to-one except at the double points, called crossings, where the shadow of the link crosses itself once transversely and at each crossing, to be able to recreate the original link, the over-strand must be distinguished from the under-strand. A link is trivial if it admits a link diagram without a crossing. A link is composite if it is a non-trivial link which cannot be written as a connected sum of two non-trivial links. A link is prime if it is not composite. A link diagram $D_{L}$ is alternating if the crossings alternate under, over, under, over, as you travel along each component of the link. A link $L$ is alternating if it has an alternating diagram $D_{L}$. Many of the knots with crossing number less than 10 are alternating, the simplest non-alternating prime knots have 8 crossings, in fact there are exactly three such knots. Alternating links have been a centerpiece of the research of knots and links. Because of their rigid structure, alternating links behave as good as one can expect. One of geometric classifications of knots is to classify the knots by the geometries of their complements; hyperbolic, torus or satellite links [3]. Menasco has shown that prime alternating knots are either hyperbolic or torus knots [13].

A link diagram $D_{L}$ is an almost alternating diagram if one crossing change makes the diagram alternating. A link $L$ is almost alternating if it is not alternating and there is an almost alternating diagram. Menasco's result has been generalized by Adams that prime almost alternating knots are either

Received March 7, 2011; Accepted November 14, 2011.

2000 Mathematics Subject Classification. Primary 57M27; Secondary 57M25, 57R56.

Corresponding author: Dongseok Kim. 
hyperbolic or torus knots [2]. It is known that no satellite knot is an almost alternating knot [9]. Authors previously showed all classical pretzel knots are either alternating or almost alternating [12]. Consequently, it provided that all classical pretzel knots are hyperbolic except a complete list of pretzel knots which are torus knots. However, to determine almost alternativity of a given link is not easy. In the present article, we first show that the determinant, signature and Alexander polynomial can not detect the almost alternativity of links.

The concept of the almost alternativity can be generalized as follows. An $m$-alternating link is a link that has a diagram in which $m$ crossing changes make it alternating, and has no diagram which can be made alternating with fewer crossing changes. In particular, 1-alternating links are precisely almost alternating links. However, instead of a fixed diagram with a simultaneous crossing changes, we might allow link isotopies in the process between link diagrams of an alternating link and the link. Kawauchi called the minimum number of crossing changes to deform $L$ to an alternating link the alternation number of the link $L$, denoted by $\operatorname{alt}(L)$, in fact, he defined it for much general setting for two arbitrary links, named Gordian distance [11]. Contrarily, we say the dealternating number of link $L$ is $m$ if $L$ is an $m$-alternating link, denoted by dalt $(L)$. It is easy to observe that $\operatorname{alt}(L) \leq \operatorname{dalt}(L)$, however, there do exists a link with a strict inequality. Recently, a series of articles has shown many interesting results. Abe found new lower bounds applying Livingston's result to Rasmussen $s$-invariant, Ozsvath-Szabo $\tau$-invariants and signatures and proves that only almost alternating torus knots are $(3,4)$ and $(3,5)$ torus knots [1]. She also find infinitely many knots which hold proper inequality. Abe and Kishimoto found the dealternating number and alternation number of some of closed 3-braids [4]. Kanenobu found the alternation number of a few torus knot [10].

\section{Main results}

One of original goals of our research on almost alternating knots was to find a knot invariant which can detect almost alternativity of knots. However, it was not successful and we find that the determinant, signature and Alexander polynomial can not detect the almost alternativity of knots in the rest of section.

\subsection{Determinant}

We find that for a given any odd integer $2 k+1$, there are infinitely many almost alternating pretzel knots whose determinant is $|2 k+1|$. Let us briefly review about the pretzel links. Let $L\left(p_{1}, p_{2}, \ldots, p_{n}\right)$ be an $n$-pretzel link in $\mathbb{S}^{3}$ where $p_{i} \in \mathbb{Z}$ represents the number of half twists as depicted in Figure 1. In particular, if $n=3$, it is called a classical pretzel link, denoted by $L(p, q, r)$.

For a given link $L$, we can find a Seifert surface $F$, an orientable surface whose boundary is $L$. Let $a_{1}, a_{2}, \ldots, a_{n}$ be a basis for the first homology 


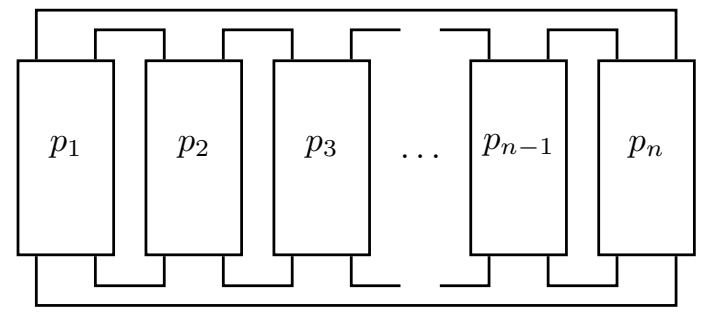

FiguRE 1. An $n$-pretzel link $L\left(p_{1}, p_{2}, \ldots, p_{n}\right)$

groups of the complement of $L, H_{1}\left(\mathbb{S}^{3}-L\right)$. A Seifert matrix $V_{L}$ of $L$ is an $n \times n$ matrix whose $(i, j)$ entry is the link number between $a_{i}$ and $a_{j}^{+}$ which is obtained from $a_{j}$ by pushing slightly off $F$ in the positive normal direction. The determinant of a link is the determinant of the Seifert matrix of $V_{L}+V_{L}^{t}$. The Alexander polynomial of link $L, \Delta_{L}(t)$ is the determinant of $V_{L}-t V_{L}^{t}$. Thus, the determinant of a knot $K$ is equal to $\Delta_{K}(-1)$ where $\Delta_{K}(t)$ is the Alexander polynomial of $L$. Since the Alexander polynomial can be obtained from the Conway polynomial as $\Delta_{L}\left(t^{2}\right)=\Lambda_{L}\left(t-t^{-1}\right)$. Using the result about the Conway polynomialss of pretzel links [12], we can easily find that the determinant of an $n$-pretzel link $L\left(p_{1}, p_{2}, \cdots, p_{n}\right)$ is $\mid \sum_{i=1}^{n} p_{1} p_{2} \cdots \hat{p}_{i}$ $\cdots p_{n} \mid$, where $\hat{p}_{i}$ is deleted in the summation. In particular, the determinant of a classical pretzel link $L(p, q, r)$ is $|p q+p r+q r|$. In the following examples, we find that for a given any odd integer $2 k+1$, there are infinitely many almost alternating pretzel knots whose determinant is $|2 k+1|$. Therefore, the determinant can not detect the almost alternativity.

Example 2.1. For each $p, K(p, 1-p,(2 k+1)+p(p-1))$ has determinant $|2 k+1|$.

Example 2.2. For each $p, K\left(p,-(p-1), 1+p(p-1), 1+p(p-1)+p^{2}(p-1)^{2}\right.$, $\left.(2 k+1)+p(p-1)+p^{2}(p-1)^{2}+\left(p(p-1)+p^{2}(p-1)^{2}\right)^{2}\right)$ has determinant $|2 k+1|$. Inductively the $(2 l+1)$-pretzel knot $K\left(p_{1}, p_{2}, \cdots, p_{2 l-1}, 1-\prod_{i=1}^{2 l-1} p_{i}\right.$, $\left.2 k+1-\prod_{i=1}^{2 l-1} p_{i}+\left(\prod_{i=1}^{2 l-1} p_{i}\right)^{2}\right)$ has determinant 1 , where $K\left(p_{1}, p_{2}, \cdots, p_{2 l-1}\right)$ has determinant 1 .

\subsection{Signature}

There are several ways to find the signature of a given knot. One can use the Seifert matrix of the knot. But, this matrix might be too big for some pretzel knots (actually this is true once we have an even $p_{i}$ ). One effective way was introduced by Gordon and Litherland by using the Goeritz matrix and $\mu(D)[8]$. Using this method, we can find the signature of pretzel knots of $K(p, q, r)$ and $K(2 l, q, r)$ where $p, q, r$ are odd. 


\section{Proposition 2.3.}

$$
\begin{gathered}
\sigma(K(p, q, r))=\left\{\begin{array}{cl}
2 & \text { if } p q+p r+q r>0 \text { and } p+q+r>0, \\
0 & \text { if } p q+p r+q r<0, \\
-2 & \text { if } p q+p r+q r>0 \text { and } p+q+r<0 .
\end{array}\right. \\
\sigma(K(2 l, q, r))=\left\{\begin{array}{cc}
-q-r & \text { if } 2 l(q+r)+q r<0, \\
-q-r+2 & \text { if } 2 l(q+r)+q r>0 \text { and } 2 l+q+r>0, \\
-q-r-2 & \text { if } 2 l(q+r)+q r>0 \text { and } 2 l+q+r<0 .
\end{array}\right.
\end{gathered}
$$

For $n$-pretzel knots, we will only look at $K\left(2 k_{1}+1,2 k_{2}+1, \cdots, 2 k_{n}+1\right)$ for the rest of the subsection. To find the signature we will use a Seifert surface $F$ but we will choose the one which consists of two discs with twisted bands. From it we choose $n-1$ natural generators of $H_{1}(F)$ which run along adjacent bands. The Seifert matrix $V_{K\left(2 k_{1}+1,2 k_{2}+1, \cdots, 2 k_{n}+1\right)}$ of $K\left(2 k_{1}+1,2 k_{2}+1, \cdots, 2 k_{n}+1\right)$ is

$$
\left(\begin{array}{cccccc}
k_{1}+k_{2}+1 & k_{2}+1 & 0 & \cdots & 0 & 0 \\
k_{2} & k_{2}+k_{3}+1 & k_{3}+1 & \cdots & 0 & 0 \\
0 & k_{3} & k_{3}+k_{4}+1 & \cdots & 0 & 0 \\
: & : & : & : & : & : \\
0 & 0 & 0 & \cdots & k_{n-2}+k_{n-1}+1 & k_{n-1}+1 \\
0 & 0 & & \cdots & k_{n-1} & k_{n-1}+k_{n}+1
\end{array}\right)
$$

Recall that the signature of a knot $K$ is $r-s$ where $r, s$ are the number of positive, negative eigenvalues of $\left(V_{K}\right)^{t}+V_{K}$, respectively. The following examples illustrate that the signature can not detect the almost alternativity.

Example 2.4. For each $l, K(-l-m, l,-l+m)$ has signature 0, and $K(-l+$ $m, l,-l+m)$ has signature 2 for all $m \geq 2$.

Example 2.5. For each $2 l$, the $(2 l+1)$-pretzel knot $K(m,-m, m,-m, \cdots,-m$, $m)$ and the $2 k$-pretzel knot $K(2,1,1, \cdots, 1,-2 l-2-($ sign of $l) 1)$ have signature $2 l$ for all $m \geq 3, k>1$.

\subsection{Alexander Polynomial}

For an almost alternating knot, we can see that its polynomial is obtained from an alternating knot and another alternating link in the skein relation. So we might expect that the polynomial of an almost alternating knot might have some properties analogous to those for alternating knots. For example, the Alexander polynomials of alternating knots are alternative and symmetric where an integral polynomial $p(t)=\sum_{i=0}^{m} c_{i} t^{i}$ is alternative if 1) all the $c_{i}$ are nonzero and 2) $\operatorname{sign}\left(c_{i}\right)=-\operatorname{sign}\left(c_{i+1}\right)$ for all $i=1,2, \cdots, m-1$ and an integral polynomial is symmetric if $t^{m} p(1 / t)=p(t)$. However, we remark that this is not a sufficient condition i.e., there are a lot of knots with alternative Alexander polynomials which are not alternating. In fact, there are only 3 knots up to 10 crossings whose Alexander polynomials are not alternative. Moreover, R. H. 
Fox conjectured that the Alexander polynomial of an alternating knot $\Delta_{K}(-t)$ has the following property, called trapezoidal [7]. An integral polynomial $p(t)=$ $\sum_{i=0}^{m} c_{i} t^{i}$ is trapezoidal if 1$)$ all the $c_{i}$ are nonzero and have the same sign, 2) $\left.t^{m} p(1 / t)=p(t), 3\right)\left|c_{0}\right| \leq\left|c_{1}\right| \leq \cdots \leq\left|c_{k}\right|$ where $k=[m / 2]$ and 4) if $c_{i}=c_{i+1}, c_{i}=c_{j}$ for all $i \leq j \leq k$. For alternating links, we can use $t^{1 / 2} \Delta_{L}(-t)$ for Fox's conjecture. It is known that Fox's conjecture is true for alternating algebraic knots [14].

If we assume that Fox's conjecture is true for all alternating links, to show that a knot is not almost alternating we might show that its Alexander polynomial is not the skein sum of $\Delta_{K^{\prime}}, \Delta_{L}$ where $\Delta_{K^{\prime}}(-t), \Delta_{L}(-t)$ are trapezoidal. It is not difficult to see that degree $\Delta_{L}=$ degree $\Delta_{K^{\prime}}-1$ by the result of $\mathrm{R}$. Crowell [6]. If we don't assume Fox's conjecture, it is easy to see that the above sentence is nonsense $i . e$, we can always find a pair of integral polynomials which satisfy the previous conditions.

Up to 11 crossings there are three knots and two links for which we can't decide whether they are almost alternating or not. We will find the Alexander polynomials of these knots $K(a), K(b), K(c)$ to get some information.

$$
\begin{aligned}
& \Delta_{K(a)}(t)=t^{6}-t^{5}-t^{4}+3 t^{3}-t^{2}-t+1 \\
& \Delta_{K(b)}(t)=t^{6}+t^{5}-6 t^{4}+9 t^{3}+-6 t^{2}+t+1 \\
& \Delta_{K(c)}(t)=-t^{6}+4 t^{5}-4 t^{4}+3 t^{3}-4 t^{2}+4 t-1
\end{aligned}
$$

We can see that none of these Alexander polynomials of $-t$ are trapezoidal. Unfortunately, we can find some polynomials which contradict our expectation.

$$
\begin{gathered}
t^{6}-t^{5}-t^{4}+3 t^{3}-t^{2}-t+1=\left[2 t^{6}-4 t^{5}+7 t^{4}-9 t^{3}+7 t^{2}-4 t+2\right] \\
+\left(t^{-1 / 2}-t^{1 / 2}\right) t^{1 / 2}\left[t^{5}-2 t^{4}+6 t^{3}-6 t^{2}+2 t-1\right] \\
t^{6}+t^{5}-6 t^{4}+9 t^{3}-6 t^{2}+t+1=\left[2 t^{6}-4 t^{5}+6 t^{4}-7 t^{3}+6 t^{2}-4 t+2\right] \\
+\left(t^{-1 / 2}-t^{1 / 2}\right) t^{1 / 2}\left[t^{5}-4 t^{4}+8 t^{3}-8 t^{2}+4 t-1\right] \\
-t^{6}+4 t^{5}-4 t^{4}+3 t^{3}-4 t^{2}+4 t-1=\left[t^{6}-2 t^{5}+5 t^{4}-7 t^{3}+5 t^{2}-2 t+1\right] \\
+\left(t^{-1 / 2}-t^{1 / 2}\right) t^{1 / 2}\left[2 t^{5}-4 t^{4}+5 t^{3}-5 t^{2}+4 t-2\right]
\end{gathered}
$$

Now, to show that such polynomials exist for all almost alternating knots we have to solve the following integral equation keeping the trapezoidal condition. Recall that the degree of two Alexander polynomials of two alternating links are related as in the equation because the equality in the equation 1 holds for alternating links as we mentioned in the beginning of this section.

$$
\begin{aligned}
& \left(a_{1}, a_{2}, a_{3}, \cdots, a_{k}, a_{k+1}, a_{k}, \cdots, a_{3}, a_{2}, a_{1}\right) \\
= & \left(b_{1},-b_{2}, b_{3}, \cdots,(-1)^{k-1} b_{k},(-1)^{k} b_{k+1},(-1)^{k-1} b_{k}, \cdots, b_{3},-b_{2}, b_{1}\right)
\end{aligned}
$$




$$
\begin{aligned}
& +\left(c_{1},-c_{2}, c_{3}, \cdots,(-1)^{k-1} c_{k},(-1)^{k} c_{k},(-1)^{k-1} c_{k-1}, \cdots, c_{2},-c_{1}, 0\right) \\
& +\left(0,-c_{1}, c_{2}, \cdots,(-1)^{k-1} c_{k-1},(-1)^{k} c_{k},(-1)^{k-1} c_{k}, \cdots, c_{3},-c_{2}, c_{1}\right)
\end{aligned}
$$

Theorem 2.6. The equation 1 has a solution which satisfies the trapezoidal condition.

Proof. The equation can be represented this way : $a_{1}=b_{1}+c_{1}, a_{2}=-\left(b_{2}+c_{1}+\right.$ $\left.c_{2}\right), a_{3}=b_{3}+c_{2}+c_{3}, \cdots, a_{k}=(-1)^{k-1}\left(b_{k}+c_{k-1}+c_{k}\right), a_{k+1}=(-1)^{k}\left(b_{k+1}+\right.$ $2 c_{k}$ ). We add the following conditions to ensure that the trapezoidal condition holds : 1) $\left|b_{i}\right| \leq\left|b_{j}\right|,\left|c_{i}\right| \leq\left|c_{j}\right|$ for all $\left.i<j, 2\right)$ if $b_{i}=b_{i+1}, b_{i}=b_{j}$ for all $i \leq j \leq k+1$, if $c_{i}=c_{i+1}, c_{i}=c_{j}$ for all $i \leq j \leq k$, and either 3) $c_{i}>0>b_{j}$ or 4) $b_{i}>0>c_{j}$. Note that these are sufficient conditions for the trapezoidal condition. We will prove that there are at least two solutions, which satisfy conditions 1),2),3) and 1),2),4), respectively. Induct on $k$. For $k=0$, let $b_{k+1}=a_{k+1}$. For $n=1$, we get $b_{2}=b_{1}-\left(2 a_{1}+a_{2}\right)$ by eliminating $c_{1}$. Thus, we have solutions $b_{2}>b_{1}>0>c_{1}, b_{2}^{\prime}>b_{1}^{\prime}>0>c_{1}^{\prime}$ for sufficiently large $b_{1},-b_{1}^{\prime}>0$. Now we assume for $k=n$, if we take $c_{n-1}=c_{n}$ then by the induction hypothesis we have two solutions which satisfy above conditions and the first $k$ equations.

All we need to show is to make $b_{k+1}$ satisfy the given condition. We notice that we have infinitely many solutions from a given solution by increasing the absolute values of the $b_{i}$ 's and $c_{j}$ 's by 1 for $b_{1}, c_{j}$, by 2 for $b_{i}, i \neq 1$. Also after fixing $c_{n-1}$, we can make the similar changes for $c_{n}, b_{n}$ and $b_{n+1}$. If $\left|b_{n}\right|>\left|b_{n+1}\right|$, we consider the two cases : 1) $b_{n}, b_{n+1}$ have the same sign, 2) $b_{n}, b_{n+1}$ have different signs. If $b_{n}, b_{n+1}$ have the same sign, then we have a solution by increasing the absolute values of $c_{n}, b_{n}$ and $b_{n+1}$ by 1 for $c_{n}, b_{n}$ and by 2 for $b_{n+1}$. So eventually we find new $c_{n}, b_{n}$ and $b_{n+1}$ satisfying all conditions. If $b_{n}, b_{n+1}$ have different signs, without loss of generality we assume that $c_{n}, b_{n+1}>0>b_{n}$. But increasing $c_{n}$ by 1 will decrease $b_{n}$ by 1 and $b_{n+1}$ by 2 , thus it reduces to the first case and it stops at finite steps. This completes the proof of the theorem.

Acknowledgements The $\mathrm{T}_{\mathrm{EX}}$ macro package PSTricks [15] was essential for typesetting the equations and figures.

\section{References}

[1] T. Abe, An estimation of the alternation number of a torus knot, J. Knot Theory Ramifications 18(3) (2009), 363-379.

[2] C. Adams, Almost alternating links, Topology and its applications 46 (1992), 151-165.

[3] C. Adams, The knot book, W. H. Freeman and Company. 1994.

[4] T. Abe and K. Kishimoto, The dealternation number and the alternation number of a closed 3-braid, J. Knot Theory Ramifications 19(9) (2010), 1157-1181.

[5] J. Conway, An enumeration of knots and links, and some of their algebraic properties, Computational problemas in abstract algebra, Pergamon Press, 1969.

[6] R. Crowell, Genus of alternating link types, Ann. of Math. 69 (1959), 258-275.

[7] R. H. Fox, Some problems in knot theory, Prentice-Hall, 1962. 
[8] C. McA. Gordon and R. Litherland, On the signature of a link, Invent. Math. 47 (1987), 53-69.

[9] J. Hoste, M. Thistlethwaite and J. Weeks, The First 1701936 Knots, Math. Intell. 20 (1998), 33-48.

[10] T. Kanenobu, Upper bound for the alternation number of a torus knot, Topology Appl., in Press.

[11] A. Kawauchi, On alternation numbers of links, Topology Appl., in Press.

[12] D. Kim and J. Lee, On pretzel links, Bull. of Aust. Math. Soc. 75(2) (2007), 253-271.

[13] W. Menasco, Closed incompressible surfaces in alternating knot and link complements, Topology 23 (1984), 37-44.

[14] K. Murasugi, On the Alexander polynomial of alternating algebraic knots, J. of Aus. Math. Soc. 39 (1985), 317-333.

[15] T. Van Zandt. PSTricks: PostScript macros for generic $\mathrm{T}_{\mathrm{E}} \mathrm{X}$. Available at ftp://ftp. princeton.edu/pub/tvz/.

Myung Jae Kim

Department of Mathematics, Kyonggi University, Suwon, 443-760, Korea

E-mail address: mjkim@kyonggi.ac.kr

Dong-Hee Lee

Department of Business Administration, Kyonggi University, Suwon, 443-760, KOREA

E-mail address: dhl@kgu.ac.kr

DONGSEOK KIM

Department of Mathematics, Kyonggi University, Suwon, 443-760, Korea

E-mail address: dongseok@kgu.ac.kr 